\title{
EXTREMUM PROPERTIES OF THE GENERALIZED RAYLEIGH QUOTIENT ASSOCIATED WITH FLUTTER INSTABILITY*
}

$\mathrm{BY}$

\author{
K. HUSEYIN \\ University of Waterloo \\ AND \\ R. H. PLAUT \\ Brown University
}

\begin{abstract}
The extremum properties of the generalized Rayleigh quotient related to flutter instability are investigated. It is shown that, in addition to the well-known stationary property, under certain circumstances the quotient exhibits maximum-minimum properties which are in contrast to those of the classical Rayleigh quotient. One consequence is that an approximate method of stability analysis using these results leads to a lower bound as opposed to an upper bound in the classical case. The results are applied to multiple-parameter systems and a physical interpretation is given for the generalized Rayleigh quotient, leading to the proof of a convexity theorem.
\end{abstract}

1. Introduction. The vibration or stability analysis of linear conservative systems leads to an eigenvalue problem involving a symmetric matrix pencil $A-\lambda B$. In such problems each eigenvalue can be expressed as a quotient in terms of its eigenvectors and the matrices $A$ and $B$. The classical Rayleigh quotient is obtained when the eigenvectors are replaced by arbitrary vectors in the space of the eigenvectors. It is well known that the Rayleigh quotient is stationary in the neighborhood of an eigenvector, with its stationary value given by the corresponding eigenvalue. This property forms the basis of the Rayleigh-Ritz technique for computing eigenvalues of a self-adjoint system. The Rayleigh quotient has an even more interesting extremum property: for $A$ and $B$ real and symmetric and $B$ positive definite, the value of the Rayleigh quotient necessarily lies between the smallest and largest eigenvalues of the pencil. In other words, the minimum (maximum) value attainable by the Rayleigh quotient is given by the smallest (largest) eigenvalue [1-4].

Linear nonconservative systems, on the other hand, lead to an eigenvalue problem associated with an asymmetric matrix pencil which has distinct left and right eigenvectors. In this case the generalized Rayleigh quotient can be introduced as a natural extension of the classical one, involving a quotient of bilinear forms rather than quadratic forms. If the matrix pencil is simple, it has been shown that the generalized Rayleigh

* Received August 28, 1972, revised version received December 18, 1972. This research was supported in part (K. Huseyin) by the National Research Council of Canada and in part (R. H. Plaut) by the Office of Naval Research under Grant No. NONR-N00014-67-A-0191-0009 and U.S. Army Research Office-Durham under Grant No. DA-ARO-D-31-124-71-G12S2. 
quotient has a stationary value at a pair of left and right eigenvectors and its value is given by the corresponding eigenvalue $[4,5]$. This corresponds directly to the stationary property of the classical Rayleigh quotient. Extremum properties in the asymmetric case, however, pose fundamental difficulties since the eigenvalues will, in general, be complex.

Attention in this paper is focussed on flutter instability, which may occur in nonconservative systems. After a brief description of the generalized Rayleigh quotient in Sec. 2, its application to this type of instability is described in Sec. 3. At flutter the left and right eigenvectors are related in a certain manner. Although extremum properties like those in the symmetric case cannot be derived in general, such properties do exist when there are only two degrees of freedom and both eigenvalues are real. This is shown in Sec. 4, and interestingly the behavior is opposite to that in the symmetric problem. In the flutter case, the value of the generalized Rayleigh quotient must lie outside of the eigenvalues if they have the same sign and it must lie between them if they have opposite signs. This result can be used to compute bounds on the eigenvalues. It also can be applied to obtain convexity properties for the flutter boundary in multipleparameter systems, as shown in Sec. 5. A physical interpretation of the generalized Rayleigh quotient is also given in this last section.

2. Generalized Rayleigh quotient. Consider the eigenvalue problem

$$
A q=\lambda B q
$$

where $A$ and $B$ are real $n \times n$ matrices with $B$ nonsingular and $q$ is a column vector of order $n$. This system of equations may be written as

$$
(A-\lambda B) q=0 .
$$

If $\lambda_{i}, i=1, \cdots, n$ are the eigenvalues of the matrix pencil $A-\lambda B$ and if the corresponding right eigenvectors are $u_{i}$ and the left eigenvectors are $v_{i}$, then

$$
\left(A-\lambda_{i} B\right) u_{i}=0, \quad i=1, \cdots, n,
$$

and

$$
v_{i}{ }^{\prime}\left(A-\lambda_{i} B\right)=0, \quad i=1, \cdots, n,
$$

where a prime denotes transpose. Eq. (2.4) is sometimes written in the form

$$
\left(A^{\prime}-\lambda_{i} B^{\prime}\right) v_{i}=0, \quad i=1, \cdots, n,
$$

and referred to as the adjoint system to Eq. (2.3). If Eq. (2.3) is premultiplied by $v_{i}{ }^{\prime}$ one obtains the scalar equations

$$
v_{i}{ }^{\prime}\left(A-\lambda_{i} B\right) u_{i}=0, \quad i=1, \cdots, n,
$$

or, in the notation to be used here,

$$
\left\langle v_{i},\left(A-\lambda_{i} B\right) u_{i}\right\rangle=0, \quad i=1, \cdots, n .
$$

Therefore the eigenvalues $\lambda_{i}$ satisfy the relations

$$
\lambda_{i}=\frac{\left\langle v_{i}, A u_{i}\right\rangle}{\left\langle v_{i}, B u_{i}\right\rangle}, \quad i=1, \cdots, n,
$$

assuming $v_{i}$ and $u_{i}$ are such that the denominator is not zero. 
The generalized Rayleigh quotient $R(x, y)$ is defined by

$$
R(x, y)=\frac{\langle y, A x\rangle}{\langle y, B x\rangle}
$$

where $x$ and $y$ are vectors of order $n$. If the matrix pencil $A-\lambda B$ is simple, so that it has $n$ linearly independent right eigenvectors, then $u_{i}$ and $v_{i}$ can be chosen to satisfy the bi-orthogonality relations

$$
\left\langle v_{i}, B u_{i}\right\rangle=\delta_{i i}, \quad i, j=1, \cdots, n,
$$

where $\delta_{i j}$ is the Kronecker delta, and $R(x, y)$ has a stationary value at $x=u_{i}, y=v_{i}$ with $R\left(u_{i}, v_{i}\right)=\lambda_{i}[5]$.

3. Flutter instability. The problem treated in this paper arises in the study of the stability of nonconservative systems. Consider a linear autonomous dynamical system with $n$ degrees of freedom. The equations of motion are assumed to be of the form

$$
M \frac{d^{2} r}{d t^{2}}+(U-\eta E) r=0
$$

where $t$ denotes time, $r(t)$ is a vector of order $n$ representing the generalized coordinates, $\eta$ is a loading parameter, and $M, U$ and $E$ are real $n \times n$ matrices with $M$ and $U$ symmetric and positive definite and $E$ nonsingular. For harmonic motion of amplitude $q$ and frequency $\omega$, one obtains

$$
(U-\eta E-\Omega M) q=0
$$

where $\Omega=\omega^{2}$. This is a double eigenvalue problem involving the loading parameter $\eta$ and the frequency parameter $\Omega$.

In a vibration analysis one is interested in the frequencies and the modes of vibration for a fixed value of $\eta$. With the notation of Sec. 2, one would then treat $\lambda=\Omega, A=$ $U-\eta E$, and $B=M$. Eq. (2.8) gives the values $\Omega_{i}$ of the squares of the vibration frequencies. At $\eta=0$ these values are real and positive and are assumed to be distinct. As the loading parameter is increased or decreased from zero, instability may occur by divergence, when one $\Omega_{i}$ value becomes zero, or by flutter, when two $\Omega_{i}$ values coalesce. If $u_{1}$ and $v_{1}$ are the eigenvectors corresponding to the equal pair of eigenvalues, they satisfy the relation

$$
\left\langle v_{1}, M u_{1}\right\rangle=0
$$

[6, 7]. Hence this matrix pencil associated with flutter is defective [5]. This is significant since the mathematical theory related to matrix pencils has been developed mostly with regard to simple (or non-defective) pencils.

In a stability analysis the loading parameter $\eta$ is treated as the eigenvalue. For this case consider $\lambda=\eta, A=U-\Omega M$, and $B=E$, and the generalized Rayleigh quotient

$$
R(x, y ; \Omega)=\frac{\langle y,(U-\Omega M) x\rangle}{\langle y, E x\rangle} .
$$

Since our attention is on flutter instability, only pairs of vectors $x, y$ which satisfy the flutter condition

$$
\langle y, M x\rangle=0
$$


will be considered in what follows. The generalized Rayleigh quotient then reduces to the form

$$
R(x, y)=\frac{\langle y, U x\rangle}{\langle y, E x\rangle}
$$

Consider a critical flutter point at which $\Omega=\Omega_{c}, \eta=\eta_{1}$, and the eigenvectors $u_{1}$ and $v_{1}$ satisfy condition (3.3). At this fixed frequency Eqs. (2.3) and (2.4) become

$$
\left(U-\Omega_{c} M-\eta_{i} E\right) u_{i}=0, \quad i=1, \cdots, n,
$$

and

$$
v_{i}{ }^{\prime}\left(U-\Omega_{c} M-\eta_{i} E\right)=0, \quad i=1, \cdots, n,
$$

while the bi-orthogonality conditions (2.10) are given by

$$
\left\langle v_{i}, E u_{i}\right\rangle=\delta_{i i}, \quad i, j=1, \cdots, n .
$$

For the class of vectors $x, y$ under consideration, $R(x, y)$ has a stationary value at $x=u_{i}$, $y=v_{i}$ with $R\left(u_{i}, v_{i}\right)=\eta_{i}$.

With the use of Eqs. (3.3) and (3.7)-(3.9) one can obtain the following:

$$
\begin{aligned}
& \left\langle v_{1}, U u_{1}\right\rangle=\eta_{1} \text {, } \\
& \left\langle v_{s}, U u_{s}\right\rangle=\eta_{s}+\Omega_{c}\left\langle v_{s}, M u_{s}\right\rangle, \quad s=2, \cdots, n, \\
& \left\langle v_{1}, U u_{s}\right\rangle=\Omega_{c}\left\langle v_{1}, M u_{s}\right\rangle, \quad s=2, \cdots, n \text {, } \\
& \left\langle v_{s}, U u_{1}\right\rangle=\Omega_{c}\left\langle v_{s}, M u_{1}\right\rangle, \quad s=2, \cdots, n \text {. }
\end{aligned}
$$

Further, if $A(\Omega)=U-\Omega M$ is considered as a function of $\Omega$ so that $\eta$ becomes a function of $\Omega$, then the first and second derivatives of $\eta$ with respect to $\Omega$ and evaluated at $\eta=\eta_{1}$, $\Omega=\Omega_{c}$ are given by $[8,9]$

$$
\left.\frac{d \eta}{d \Omega}\right|_{\substack{\eta=\eta_{1} \\ \Omega=\Omega_{c}}}=\frac{\left\langle v_{1}, M u_{1}\right\rangle}{\left\langle v_{1}, E u_{1}\right\rangle}=0
$$

and

$$
\left.\frac{d^{2} \eta}{d \Omega^{2}}\right|_{\substack{\eta=\eta_{1} \\ \Omega=\Omega_{c}}}=2 \sum_{s=2}^{n} \frac{\left\langle v_{1}, M u_{s}\right\rangle\left\langle v_{s}, M u_{1}\right\rangle}{\left(\eta_{1}-\eta_{s}\right)}
$$

4. Extremum properties. In order to investigate extremum properties of the generalized Rayleigh quotient with respect to the flutter point $\eta_{1}$, it is convenient to write $x$ and $y$ in the form

$$
x=u_{1}+w, \quad y=v_{1}+z .
$$

When this form is substituted into Eq. (3.5) one obtains, after using Eq. (3.3),

$$
\left\langle v_{1}, M w\right\rangle+\left\langle z, M u_{1}\right\rangle+\langle z, M w\rangle=0 .
$$

Also, the generalized Rayleigh quotient defined in Eq. (3.6) can be written in the form

$$
R(x, y)=R\left(u_{1}, v_{1}\right)+\frac{\left\langle z,\left(U-\Omega_{c} M-\eta_{1} E\right) w\right\rangle}{\langle y, E x\rangle}
$$


with the use of Eqs. (3.7)-(3.10), (4.1), and (4.2). If in addition $w$ and $z$ are expressed as

$$
w=\sum_{i=1}^{n} \alpha_{i} u_{i}, \quad z=\sum_{i=1}^{n} \beta_{i} v_{i}
$$

then Eq. (4.3) becomes

$$
R(x, y)=R\left(u_{1}, v_{1}\right)+h
$$

where

$$
h=\frac{1}{\langle y, E x\rangle} \sum_{s=2}^{n}\left(\eta_{s}-\eta_{1}\right) \alpha_{s} \beta_{s} .
$$

It is seen from Eq. (4.5) that the generalized Rayleigh quotient will have a local minimum (maximum) at $u_{1}, v_{1}$ if $h \geq 0(h \leq 0)$ for all $x, y$ in a neighborhood of $u_{1}, v_{1}$. Such extremum results will now be derived for the case of $n=2$ with $\eta_{1}$ real and $\eta_{1} \neq \eta_{2}$. Since $\eta_{1}$ is real, $\eta_{2}$ is necessarily real and so are the corresponding eigenvectors, and $\alpha_{i}$ and $\beta_{i}$ in Eq. (4.4) will be taken as real numbers in what follows. It follows that $x$ and $y$ will be real.

First, it is noted that Eq. (3.15) takes the form

$$
\left.\frac{d^{2} \eta}{d \Omega^{2}}\right|_{\substack{n=\eta_{1} \\ \Omega=\Omega_{c}}}=2 \frac{\left\langle v_{1}, M u_{2}\right\rangle\left\langle v_{2}, M u_{1}\right\rangle}{\left(\eta_{1}-\eta_{2}\right)}
$$

Next consider an asymptotic analysis in which $w$ and $z$ are proportional to a small parameter $\epsilon$. One can then obtain

$$
h=\left(\eta_{2}-\eta_{1}\right) \alpha_{2} \beta_{2}
$$

from Eqs. (4.1), (4.5), and (4.6), and

$$
\left\langle v_{1}, M w\right\rangle+\left\langle z, M u_{1}\right\rangle=0
$$

from Eq. (4.2) as first-order approximations. This last expression can be written as

$$
\alpha_{2}\left\langle v_{1}, M u_{2}\right\rangle+\beta_{2}\left\langle v_{2}, M u_{1}\right\rangle=0
$$

and multiplying by $\beta_{2}\left\langle v_{2}, M u_{1}\right\rangle$ leads to the inequality

$$
\left\langle v_{1}, M u_{2}\right\rangle\left\langle v_{2}, M u_{1}\right\rangle \alpha_{2} \beta_{2} \leq 0 .
$$

The following conclusion can then be deduced from expressions (4.5), (4.7), (4.8) and (4.11): subject to condition (3.5) with real $x$ and $y$, the generalized Rayleigh quotient (3.6) has a local minimum (maximum) at $x=u_{1}, y=v_{1}$ if

$$
\left.\frac{d^{2} \eta}{d \Omega^{2}}\right|_{\substack{\eta=\eta_{1} \\ \Omega=\Omega_{c}}}>0 \quad(<0) .
$$

Inequality (4.12) can be related to the two values of $\eta_{1}$ at which flutter occurs. Let superscripts $a$ and $b$ designate the two flutter points. Then the characteristic equation

$$
\operatorname{det}|U-\Omega M-\eta E|=0
$$

has a double root in $\Omega$ at $\Omega=\Omega_{c}{ }^{a}, \eta=\eta_{1}{ }^{a}$ and at $\Omega=\Omega_{c}{ }^{b}, \eta=\eta_{1}{ }^{b}$. A typical loading-frequency 
curve for the case $0<\eta_{1}{ }^{a}<\eta_{1}{ }^{b}$ is depicted in Fig. 1. It is a conic section, and one can see that $d^{2} \eta / d \Omega^{2}$ is negative at $\eta_{1}{ }^{a}$ and positive at $\eta_{1}{ }^{b}$. The cases of eigenvalues with opposite sign and of two negative eigenvalues can be drawn similarly, and one obtains the following conclusions:

(i) if $0<\eta_{1}{ }^{a}<\eta_{1}{ }^{b}$, then $R(x, y)$ has a local maximum at $\eta_{1}{ }^{a}$ and a local minimum at $\eta_{1}{ }^{b}$;

(ii) if $\eta_{1}{ }^{a}<0<\eta_{1}{ }^{b}$, then $R(x, y)$ has a local minimum at $\eta_{1}{ }^{a}$ and a local maximum at $\eta_{1}{ }^{b}$;

(iii) if $\eta_{1}{ }^{a}<\eta_{1}{ }^{b}<0$, then $R(x, y)$ has a local maximum at $\eta_{1}{ }^{a}$ and a local minimum at $\eta_{1}^{b}$.

If one solves explicitly for $\eta_{1}{ }^{a}, \eta_{1}{ }^{b}$ and the corresponding eigenvectors in terms of the elements of the matrices $U, M$, and $E$, one can show that the generalized Rayleigh quotient must lie in one of the shaded regions shown in Fig. 2. That is, for cases (i) and (iii) $R(x, y)$ cannot lie between $\eta_{1}{ }^{a}$ and $\eta_{1}{ }^{b}$, while for case (ii) it must lie between these two eigenvalues (see Appendix A).

The extremum properties derived above are in direct contrast to those for symmetric

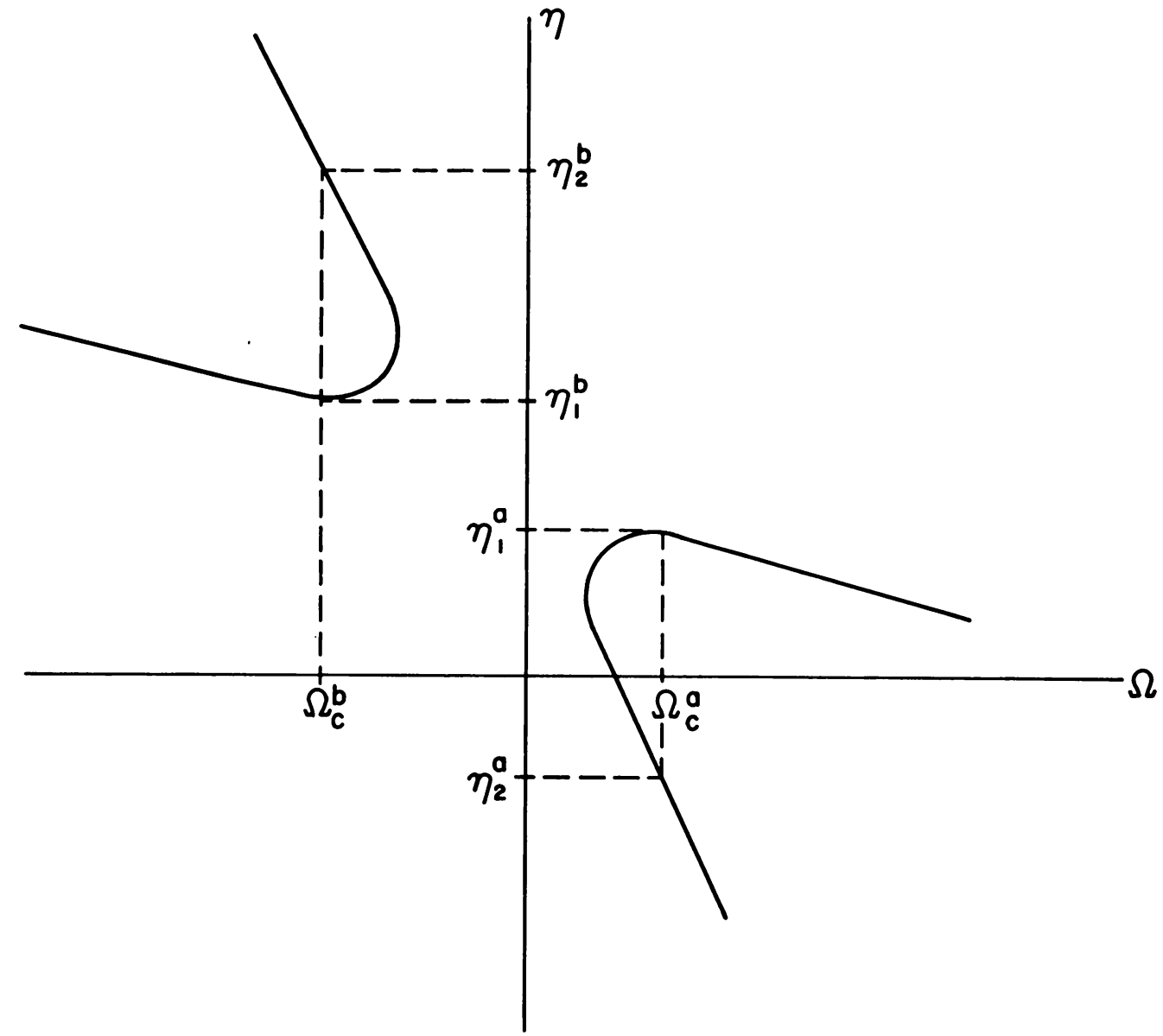

Fig. 1. Loading-frequency relationship. 
(i)

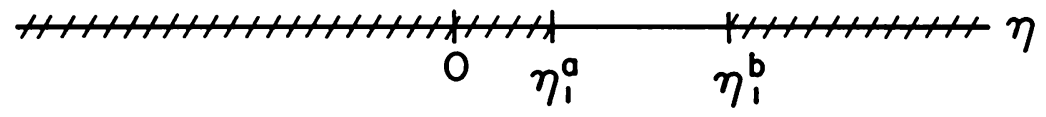

(ii)

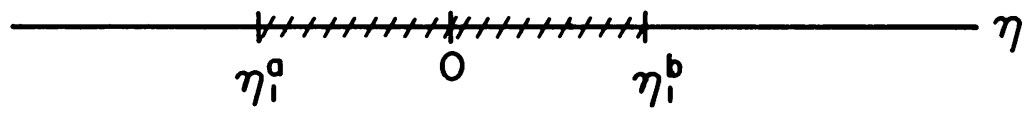

(iii)

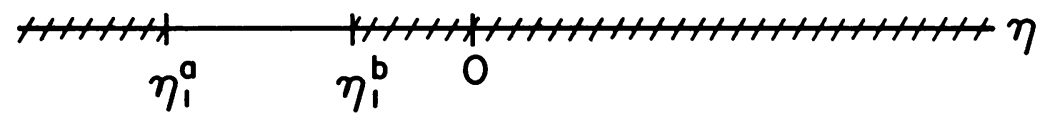

FIG. 2. Range of generalized Rayleigh quotient.

systems. If $U$ and $E$ are real symmetric matrices of order two, $U$ is positive definite, $E$ is nonsingular, and $\eta_{1}{ }^{a}, \eta_{1}{ }^{b}$ are now defined as the eigenvalues of the pencil $U-\eta E$ with $\eta_{1}{ }^{a}<\eta_{1}{ }^{b}$, then the classical Rayleigh quotient

$$
R(x)=\frac{\langle x, U x\rangle}{\langle x, E x\rangle}
$$

can only take values in the unshaded regions of Fig. 2.

As in the symmetric case, an approximate procedure can be used to compute eigenvalues and also to determine some bounds on the eigenvalues. If both critical values of $\eta$ at flutter are positive, for example, then one can obtain a lower bound for the smaller critical value and an upper bound for the higher value by using, in Eq. (3.6), vectors $x, y$ satisfying Eq. (3.5) (see Fig. 2(i)). A lower bound $\eta_{L}$ for $\eta_{1}{ }^{a}$ is especially useful since it assures that the system is stable for $\eta<\eta_{L}$. If one attempts to approach this critical value $\eta_{1}{ }^{a}$ by an iterative process, $\eta_{1}{ }^{a}$ will be approached from below according to the present results, while the Rayleigh-Ritz procedure for symmetric systems approaches the smallest critical value from above and yields an upper bound rather than a lower bound.

5. Multiple-parameter systems. The generalized Rayleigh quotient has an interesting interpretation for systems with multiple loading parameters. In addition, the results of Sec. 4 can be used to derive some convexity properties for the flutter boundary in the space of the loading parameters.

If $\eta^{k}, k=1, \cdots, m$ are independent loading parameters with associated matrices $E^{k}$, then Eq. (3.2) has the form

$$
\left(U-\sum_{k=1}^{m} \eta^{k} E^{k}-\Omega M\right) q=0
$$

and the corresponding vector $p$ in the adjoint system satisfies the equation

$$
p^{\prime}\left(U-\sum_{k=1}^{m} \eta^{k} E^{k}-\Omega M\right)=0 .
$$

Pre-multiplying Eq. (5.1) by $p^{\prime}$ leads to the relation 


$$
\left\langle p,\left(U-\sum_{k=1}^{m} \eta^{k} E^{k}-\Omega M\right) q\right\rangle=0 .
$$

Consider the $m$-dimensional loading space with coordinates $\eta^{k}$. The points in this space corresponding to flutter instability comprise the "flutter boundary". Let $q=x$, $p=y$ designate the eigenvectors at a flutter point. The flutter condition (3.5) must be satisfied, so that Eq. (5.3) becomes

$$
\langle y, U x\rangle-\sum_{k=1}^{m} \eta^{k}\left\langle y, E^{k} x\right\rangle=0 .
$$

Eq. (5.4) is then the equation of the hyperplane tangent to the flutter boundary at this point, and the normal vector to this hyperplane has components $N^{k}$ given by

$$
N^{k}=\left\langle y, E^{k} x\right\rangle
$$

(see Appendix B).

Now consider a ray emerging from the origin in the loading space. If the direction cosines are given by $l^{k}, k=1, \cdots, m$ and the distance along the ray is $\eta$, then

$$
\eta^{k}=\eta l^{k}, \sum_{k=1}^{m}\left(l^{k}\right)^{2}=1 .
$$

Also, define

$$
E=\sum_{k=1}^{m} l^{k} E^{k}
$$

and assume that the ray initially hits the flutter boundary at $\eta=\eta_{1}>0$ with corresponding eigenvectors $u_{1}, v_{1}$, and that it intersects the hyperplane defined by Eq. (5.4) at $\eta=\eta_{t}$. It follows that

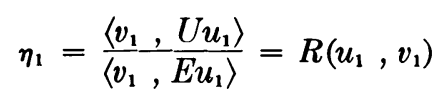

and

$$
\eta_{t}=\frac{\langle y, U x\rangle}{\langle y, E x\rangle}=R(x, y) .
$$

In other words, the generalized Rayleigh quotient $R(x, y)$ gives the distance $\eta_{t}$ from the origin to the intersection of the ray with the hyperplane, while its stationary value $R\left(u_{1}, v_{1}\right)$ is the distance $\eta_{1}$ to the flutter boundary. This is depicted in Fig. 3, where the eigenvectors $x, y$ correspond to point $A$ and $u_{1}, v_{1}$ correspond to point $B$.

Convexity properties of the flutter boundary for the case $n=2, m=2$ were investigated in [6]. These properties now can be generalized to cover an arbitrary number of loading parameters by application of the results of Sec. 4 and the interpretation above. For example, with $\eta_{1}$ representing the initial loss of stability by flutter on the positive ray, it follows from Eqs. (5.8), (5.9) and Figs. 2, 3 that

$$
\eta_{t} \leq \eta_{1}
$$

for points $A$ in the neighborhood of $B$. Hence the flutter boundary at $B$ is convex toward the origin. This result can be of practical use in obtaining lower and upper bounds on the portion of the flutter boundary which yields initial flutter instability. 


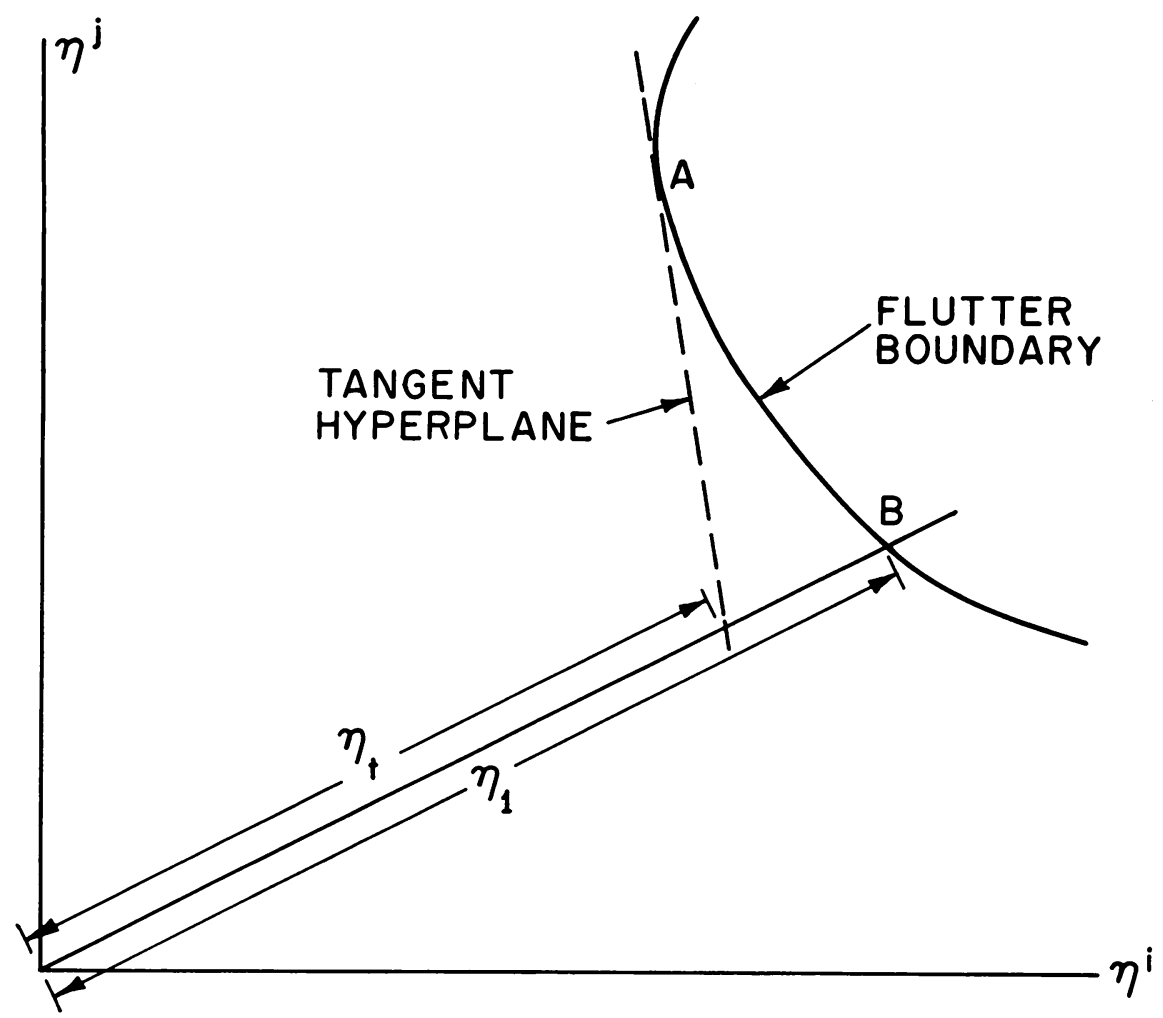

Fig. 3. Flutter boundary.

In addition one can determine conditions under which the flutter boundary consists of hyperplanes. If the eigenvectors $x$ and $y$ at a flutter point are independent of the direction cosines of the ray to that point, then the normal vector with components given by Eq. (5.5) is constant for that portion of the flutter boundary and hence this boundary is linear. For the case $n=2$ this condition is satisfied if Eq. (5.1) is in the form such that $M$ is the identity matrix and $U$ is a diagonal matrix and if the ratio of the off-diagonal terms for each matrix $E^{k}$ is the same.

For multiple-parameter conservative systems the classical Rayleigh quotient (4.14) can be interpreted as a similar distance in the loading space. The matrices $E^{k}$ are symmetric and only divergence instability can occur. Consider any values of $n$ and $m$. If $E$ is positive definite for a given ray, $R(x)$ must lie between the smallest and largest eigenvalues $\eta_{i}$. It then follows that the initial portion of the divergence boundary intersected by the ray is concave toward the origin and the final portion is convex. If $E$ is not positive definite for a ray, one can apply similar reasoning to the reciprocal of $R(x)$ and conclude that the initial portion of the divergence boundary is concave toward the origin. Also, it is easy to show that the divergence boundary consists of hyperplanes if $U$ is diagonal and if $E^{k}, k=1, \cdots, m$ are all diagonal. Previous investigations of these convexity properties for linear conservative and "pseudo-conservative" systems include [10-14].

Appendix A. The results shown in Fig. 2 will now be proved. Consider $n=2$ and $\eta_{1}{ }^{a}<\eta_{1}{ }^{b}$. Let 


$$
\eta_{1}^{\sigma} \equiv R\left(u_{1}^{\sigma}, v_{1}^{\sigma}\right), \quad \sigma=a, b,
$$

let $\eta_{\mathfrak{t}}$ be defined by Eq. (5.9) where $x, y$ satisfy Eq. (3.5), and let

$$
h^{\sigma} \equiv \eta_{t}-\eta_{1}^{\sigma}, \quad \sigma=a, b .
$$

It is desired to show that $h^{a}$ and $h^{b}$ have the same (opposite) signs whenever $\eta_{1}{ }^{a}$ and $\eta_{1}{ }^{b}$ have the same (opposite) signs. Fig. 2 then follows directly from this.

Assume that the matrices $M$ and $U$ have been simultaneously diagonalized by a linear transformation such that $M$ is the identity matrix and $U$ is a diagonal matrix. The characteristic equation (4.13) and the condition for a double root in $\Omega$ lead to the values

$$
\eta_{1}^{\sigma}=\left(U_{22}-U_{11}\right) / D^{\sigma}, \quad \sigma=a, b
$$

and

$$
\Omega_{c}^{\sigma}=\left[U_{11} E_{22}-U_{22} E_{11} \pm\left(U_{11}+U_{22}\right)\left(-E_{12} E_{21}\right)^{1 / 2}\right] / D^{\sigma}, \quad \sigma=a, b
$$

where

$$
D^{\sigma}=E_{22}-E_{11} \pm 2\left(-E_{12} E_{21}\right)^{1 / 2}, \quad \sigma=a, b
$$

with the appropriate choice of sign so that $\eta_{1}{ }^{a}<\eta_{1}{ }^{b}$. For the eigenvectors, let

$$
u_{1}{ }^{a} \equiv\left(\begin{array}{l}
\mu_{1} \\
\mu_{2}
\end{array}\right), \quad u_{1}{ }^{b} \equiv\left(\begin{array}{l}
\gamma_{1} \\
\gamma_{2}
\end{array}\right), \quad v_{1}^{a} \equiv\left(\begin{array}{l}
\nu_{1} \\
\nu_{2}
\end{array}\right), \quad v_{1}{ }^{b} \equiv\left(\begin{array}{l}
\epsilon_{1} \\
\epsilon_{2}
\end{array}\right) .
$$

With the use of Eqs. (3.7)-(3.9) and (A3)-(A5) one can calculate these values and show that

$$
\mu_{1} \gamma_{2}+\mu_{2} \gamma_{1}=0, \quad \nu_{1} \epsilon_{2}+\nu_{2} \epsilon_{1}=0,
$$

and

$$
\mu_{1} \nu_{1}=1 / D^{a}, \quad \gamma_{1} \epsilon_{1}=1 / D^{b},
$$

while the flutter condition (3.3) yields

$$
\mu_{1} \nu_{1}+\mu_{2} \nu_{2}=0, \quad \gamma_{1} \epsilon_{1}+\gamma_{2} \epsilon_{2}=0 .
$$

From Eq. (4.6),

$$
h^{\sigma}=\left(\eta_{2}{ }^{\sigma}-\eta_{1}{ }^{\sigma}\right) \alpha_{2}{ }^{\sigma}{\beta_{2}}^{\sigma} /\langle y, E x\rangle, \quad \sigma=a, b .
$$

Let the expression in Eq. (4.7) be denoted by $\kappa$, so that

$$
\kappa^{\sigma} \equiv 2\left\langle\nu_{1}^{\sigma}, M u_{2}^{\sigma}\right\rangle\left\langle v_{2}^{\sigma}, M u_{1}^{\sigma}\right\rangle /\left(\eta_{1}{ }^{\sigma}-\eta_{2}{ }^{\sigma}\right), \quad \sigma=a, b .
$$

Then it is seen from Fig. 1 that $\kappa^{a}$ and $\kappa^{b}$ have opposite signs. Eq. (A10) may be written in the form

$$
h^{\sigma}=\frac{2 \alpha_{2}{ }^{\sigma}{\beta_{2}}^{{ }}\left\langle v_{1}{ }^{\sigma}, M u_{2}{ }^{\sigma}\right\rangle\left\langle v_{2}{ }^{\sigma}, M u_{1}{ }^{\sigma}\right\rangle}{\kappa^{\sigma}\langle y, E x\rangle}, \quad \sigma=a, b,
$$

and, with the use of Eqs. (3.3), (4.1), and (4.4),

$$
h^{\sigma}=\frac{2\left\langle v_{1}^{\sigma}, M x\right\rangle\left\langle y, M u_{1}^{\sigma}\right\rangle}{\kappa^{\sigma}\langle y, E x\rangle}, \quad \sigma=a, b .
$$


Now let

$$
x \equiv\left(\begin{array}{c}
\phi_{1} \\
\phi_{2}
\end{array}\right), \quad y \equiv\left(\begin{array}{l}
\psi_{1} \\
\psi_{2}
\end{array}\right)
$$

where

$$
\phi_{1} \psi_{1}+\phi_{2} \psi_{2}=0
$$

from Eq. (3.5). Using Eqs. (A7), (A9) and (A15), one can obtain the following relations:

$$
\begin{aligned}
\left\langle v_{1}{ }^{a}, M x\right\rangle & =\nu_{1}\left(\phi_{2} \gamma_{1}+\phi_{1} \gamma_{2}\right) / \dot{\gamma}_{2} \\
\left\langle y, M u_{1}{ }^{a}\right\rangle & =\mu_{1} \psi_{1}\left(\phi_{2} \gamma_{1}+\gamma_{2} \phi_{1}\right) /\left(\phi_{2} \gamma_{1}\right) \\
\left\langle v_{1}{ }^{b}, M x\right\rangle & =\epsilon_{1}\left(\phi_{1} \gamma_{2}-\phi_{2} \gamma_{1}\right) / \gamma_{2} \\
\left\langle y, M u_{1}{ }^{b}\right\rangle & =\gamma_{1} \psi_{1}\left(\phi_{2} \gamma_{1}-\phi_{1} \gamma_{2}\right) /\left(\phi_{2} \gamma_{1}\right) .
\end{aligned}
$$

Substitution of Eqs. (A16) into (A13) and application of Eqs. (A3) and (A8) finally yield the following expressions:

$$
\begin{aligned}
& h^{a}=\eta_{1}{ }^{a} \xi\left(\phi_{2} \gamma_{1}+\phi_{1} \gamma_{2}\right)^{2} / \kappa^{a} \\
& h^{b}=-\eta_{1}{ }^{b} \xi\left(\phi_{2} \gamma_{1}-\phi_{1} \gamma_{2}\right)^{2} / \kappa^{b}
\end{aligned}
$$

where $\xi=2 \psi_{1} /\left[\phi_{2} \gamma_{1} \gamma_{2}\left(U_{22}-U_{11}\right)\langle y, E x\rangle\right]$. Since $\kappa^{a}$ and $\kappa^{b}$ have opposite signs, it is seen from Eqs. (A17) that $h^{a}$ and $h^{b}$ have the same (opposite) signs whenever $\eta_{1}{ }^{a}$ and $\eta_{1}{ }^{b}$ have the same (opposite) signs.

Appendix B. Let $\eta_{F}{ }^{k}, k=1, \ldots, m$ represent the coordinates of a flutter point $F$ in the loading space and let $x$ and $y$ denote the corresponding right and left eigenvectors, respectively. Then, from Eq. (5.1),

$$
\left(U-\sum_{k=1}^{m} \eta_{F}^{k} E^{k}-\Omega M\right) x=0 .
$$

Pre-multiplying by $y^{\prime}$ gives

$$
\left\langle y,\left(U-\sum_{k=1}^{m} \eta_{F}{ }^{k} E^{k}-\Omega M\right) x\right\rangle=0
$$

and, due to the flutter condition (3.5),

$$
\left\langle y,\left(U-\sum_{k=1}^{m} \eta_{F}{ }^{k} E^{k}\right) x\right\rangle=0
$$

It will be demonstrated that the equation

$$
\left\langle y,\left(U-\sum_{k=1}^{m} \eta_{k} E^{k}\right) x\right\rangle=0
$$

represents the tangent hyperplane to the flutter boundary at this point $F$.

First, note that Eq. (B4) does represent a hyperplane with derivatives

$$
\frac{\partial \eta^{r}}{\partial \eta^{*}} \equiv \eta^{r \cdot s}=-\frac{\left\langle y, E^{*} x\right\rangle}{\left\langle y, E^{r} x\right\rangle}
$$

in arbitrary $\eta^{r}-\eta^{s}$ planes. This hyperplane passes through the point $F$, since the values $\eta^{k}=\eta_{F}{ }^{k}$ satisfy Eq. (B4). 
Next consider the characteristic surface in the vicinity of the flutter point $F$. Critical points in the neighborhood of $F$ should satisfy Eqs. (5.1), (5.2), and the flutter condition (3.5), and the critical values of $\eta^{r}$ and $\Omega$ can, therefore, be described as functions of $m-1$ independent variables. Choosing the $\eta^{i}, j \neq r$, as these $m-1$ variables, assuming a solution in the form $\eta^{r}\left(\eta^{j}\right)$ and $\Omega\left(\eta^{j}\right)$, inserting this into Eq. (5.3) and differentiating the resulting equation with respect to an arbitrary $\eta^{j}$, say $\eta^{s}$, one obtains

$$
\begin{aligned}
\left\langle p^{\prime s},\left(U-\sum_{k=1}^{m} \eta^{k} E^{k}-\Omega M\right) q\right\rangle+\langle p,(U & \left.\left.-\sum_{k=1}^{m} \eta^{k} E^{k}-\Omega M\right) q^{\cdot s}\right\rangle \\
& -\left\langle p,\left(\eta^{r \cdot s} E^{r}+E^{*}+\Omega^{\cdot s} M\right) q\right\rangle=0
\end{aligned}
$$

where superscripts $s$ following a comma denote differentiation with respect to $\eta^{s}$. The first two terms in Eq. (B6) are zero due to Eqs. (5.1) and (5.2). Evaluating the last term at $F$ and using the flutter condition yields

$$
\eta^{r, s}=-\frac{\left\langle y, E^{s} x\right\rangle}{\left\langle y, E^{r} x\right\rangle}
$$

These derivatives for the flutter boundary at $F$ coincide with Eq. (B5), and it follows that Eq. (B4) represents the tangent hyperplane at $F$.

The components $N^{k}$ of a normal vector to the tangent hyperplane at $F$ satisfy the equation

$$
\sum_{k=1}^{m}\left(\eta_{F}{ }^{k}-\eta^{k}\right) N^{k}=0
$$

where $\eta^{k}, k=1, \cdots, m$ is any point in the hyperplane, since the dot product between the normal and any vector in the hyperplane must be zero. By subtracting Eq. (B3) from Eq. (B4) and comparing the result with Eq. (B8), it follows that the values

$$
N^{k}=\left\langle y, E^{k} x\right\rangle
$$

are the components of such a normal vector.

\section{REFERENCES}

[1] Lord Rayleigh, Theory of sound, vol. 1, Macmillan, New York, 1929; Dover, New York, 1945, sec. 88

[2] F. R. Gantmacher, The theory of matrices, vol. 1, Chelsea, New York, 1959, ch. 10

[3] S. H. Gould, Variational methods for eigenvalue problems, University of Toronto, Toronto, 1957, ch. II

[4] A. M. Ostrowski, On the convergence of the Rayleigh quotient iteration for the computation of characteristic roots and vectors, Arch. Rat. Mech. Anal. pt. I, 1, 233-241 (1957); pt. II, 2, 423-428 (1958); pt. III, 3, 325-340 (1959); pt. IV, 3, 341-347 (1959); pt. V, 3, 472-481 (1959); pt. VI, 4, 153-165 (1960)

[5] P. Lancaster, Lambda-matrices and vibrating systems, Pergamon, 1966, sec. 2.8

[6] K. Huseyin and R. H. Plaut, The elastic stability of two-parameter nonconservative systems, J. Appl. Mech. 40, 175-180 (1973)

[7] R. H. Plaut, Determining the nature of instability in nonconservative problems, AIAA J. 10, 967-968 (1972)

[8] P. Lancaster, On eigenvalues of matrices dependent on a parameter, Num. Math. 6, 377-387 (1964)

[9] R. H. Plaut and K. Huseyin, Derivatives of eigenvalues and eigenvectors in non-self-adjoint systems, AIAA J. 11, 250-251 (1973)

[10] P. F. Papkovich, Works on the structural mechanics of ships, vol. 4, Moscow, 1963 (in Russian) 
[11] J. D. Renton, Buckling of frames composed of thin-wall members, in A. H. Chilver, editor, Thinwalled structures, Wiley, New York, 1967, pp. 1-59

[12] K. Huseyin and J. Roorda, The loading-frequency relationship in multiple eigenvalue problems, J. Appl. Mech. 38, 1007-1011 (1971)

[13] K. Huseyin and H. Leipholz, Divergence instability of multiple-parameter circulatory systems, Quart. Appl. Math. 31, 185-197 (1973)

[14] F. Buckens, Über Eigenwertscharen, Öst. Ing. Arch. 12, $82-93$ (1958) 